\title{
Application of retrograde dissection method for isolation of bone marrow cells from rat femurs and tibiae
}

\author{
C.M. Li*, B.M. Fu*, L.C. Zhang, B. Tang, L. Zhu, Y. Zhao and J. Zhang \\ Hepatobiliary and Pancreatic Surgery Ward, \\ Second Affiliated Hospital of Kunming Medical University, Kunming, China \\ *These authors contributed equally to this study. \\ Corresponding authors: B.M. Fu / J. Zhang \\ E-mail: fubimang_bm@163.com / zhanjie_zj@163.com
}

Genet. Mol. Res. 15 (2): gmr.15028178

Received December 2, 2015

Accepted January 15, 2016

Published May 25, 2016

DOI http://dx.doi.org/10.4238/gmr.15028178

\begin{abstract}
Currently, there is no practical and efficient method for the isolation of bone marrow cells (BMCs) from rat femurs and tibiae. Here, we attempted to develop a rapid, simple, effective, and non-contaminating method for the isolation of BMCs from rat femurs and tibiae. Rat femurs and tibiae were dissected from the ankle to the hip joint; subsequently, a three-step "locate-slide-twist" procedure was performed using scissors and forceps to remove the femurs and tibiae completely, from the surrounding musculature. The bones were flushed with phosphate-buffered saline to harvest BMCs. The femurs and tibiae were dissected in $1.8 \pm 0.6 \mathrm{~min}$, and the BMC suspension preparation time was $13.1 \pm 2.3 \mathrm{~min}$. The bone marrow cavities did not incur any fractures or injuries during the isolation. Culture of harvested BMCs for $72 \mathrm{~h}$ led to a significant increase in cell number from $4.4 \pm 0.3 \times 10^{6}$ to $6.9 \pm 0.7 \times 10^{6}(\mathrm{P}<0.01)$ with no significant decrease in viability $(98.1 \pm 0.6 \%$ vs $96.2 \pm 1.1 \%$; P > 0.05). Microscopic examination of the isolated BMCs after the 72-h incubation period revealed the no-microbial or muscle cell contamination. Furthermore, flow cytometry revealed that cultured BMCs (72-h culture)
\end{abstract}


grew well. Here, we have reported a rapid, simple, effective, and noncontaminating method for the isolation of BMCs from rat femurs and tibiae by using retrograde dissection. This method can be used to harvest a large number of viable BMCs without the risk of contamination from muscle and connective tissues.

Key words: Retrograde dissection; Tibiae; Femurs; Rat; Bone marrow cells

\section{INTRODUCTION}

Harvesting bone marrow cells (BMCs) from murine femurs and tibiae is the first step towards conducting research into bone marrow-derived cells such as dendritic cells and mesenchymal stem cells. The method used to isolate cells from rat femurs and tibiae directly affects the ischemic time of BMCs; despite this, the existing literature does not detail any reports or convenient standard methods for this type of isolation. The small size and fragile nature of the bones of rat hind legs present unique challenges during the isolation of BMCs. Additionally, the strong surrounding muscles, as well as their firm attachments, make it difficult to obtain clean femurs and tibiae for the isolation of BMCs. Currently available literature describes methods that make use of surgical blades, gauze, or Kleenex tissues to facilitate the removal of muscle tissues from rat hind limbs (Talmor et al., 1998; Lutz et al., 1999; Soleimani and Nadri, 2009; Li et al., 2013; Davis, 2013; Smajilagić et al., 2013). However, our application of the methods suggested in these studies resulted in incomplete removal of the muscle, bone fracture, and contamination of isolated BMCs. Here, we report a rapid, effective, simple, and non-contaminating method for the isolation of BMCs from rat femurs and tibiae by retrograde dissection, based on the anatomic relationship among the bones of the rat hind leg and the adjacent muscle and connective tissues.

\section{MATERIAL AND METHODS}

\section{Materials}

\section{Rats}

Male Wistar rats $(200 \pm 20 \mathrm{~g})$ were purchased from the Experimental Animal Center of Sun Yat-sen University. The experimental protocol was approved by the institutional Animal Care Committee; all animals received humane care in compliance with the Principles of Laboratory Animal Care.

\section{Reagents}

Penicillin, streptomycin, phosphate buffered saline (PBS) solution, and RPMI1640 medium were purchased from Gibco (Thermo Fisher Scientific, Waltham, MA, USA). Fetal bovine serum (FBS) was purchased from HyClone (GE Healthcare, Little Chalfont, UK). Cell culture dishes $(10 \mathrm{~cm})$ and culture flasks $\left(25 \mathrm{~cm}^{2}\right)$ were obtained from Corning (Corning, NY, USA). Trypan blue was provided by the Medical Immunological Lab of Sun Yat-Sen University (Guangzhou, China). 


\section{Methods}

\section{Dissection of hind limbs}

Euthanized rats were rinsed with $70 \%$ ethanol for $5 \mathrm{~min}$. The following procedures were performed using aseptic techniques under a vertical laminar flow hood equipped with a UV light. A $2 \mathrm{~mm}$ incision was made approximately $2 \mathrm{~mm}$ above the urethral orifice toward the lateral flank of the thigh. The abdominal skin was pulled downward and upward to expose the abdominal wall without impairing the integrity of the peritoneal cavity. The skin of the anterior surface of both the hind limbs was dissected downward to expose the dorsum pedis. The leg was fixed using a pair of forceps, and a pair of scissors was placed between the bone and skin at the ankle joint space where the joint was transected. The skin of the posterior surface of the hind limb was preserved for better fixation (Figure 1).

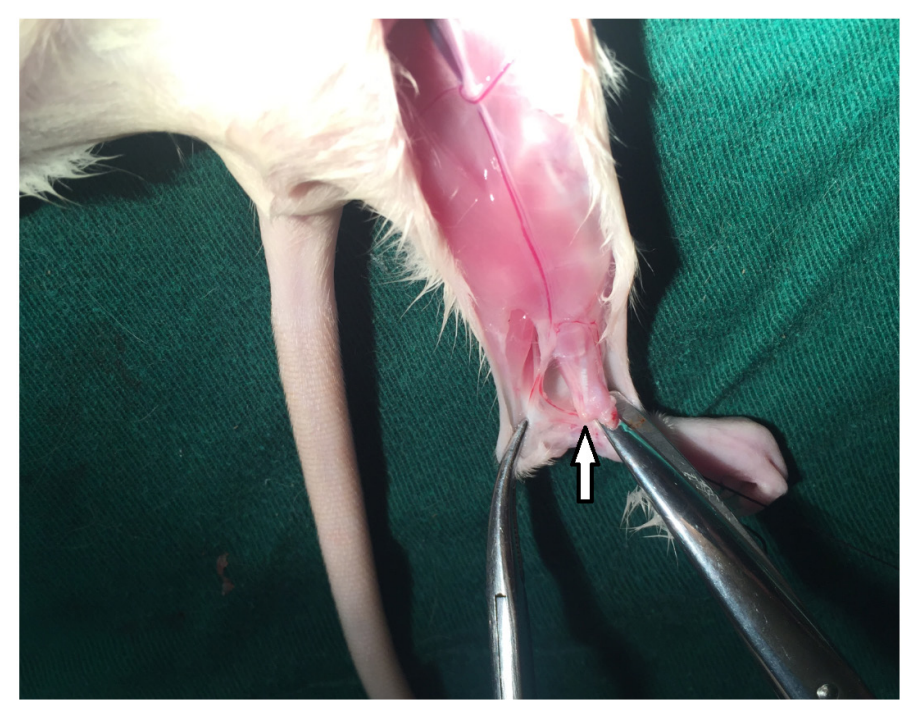

Figure 1. Isolation of femur and tibia; ankle joint was transected (arrow).

\section{Isolation of tibiae}

The Achilles tendons were immobilized and transected (Figure 2A). The distal end of the tibia was isolated and cut within the ankle joint cavity. The tibia capitulum was held by a pair of forceps, one third of the scissor blades were crossed on the distal end of the tibia, and the scissors were "slid" toward the proximal end of the tibia upon the surfaces of the tibia and fibula to expose the tibia up to the level of the knee joint (Figure 2B and C). The muscles were retracted over the knee joint; the femur was held by a pair of scissors at the proximal end of the knee joint space and gently twisted toward the reversed genuflex of the knee joint (that is, twisted against the direction of motion of the knee joint; Figure 2D). At this point, the entire proximal end of the tibia (knee joint end) was popped out and completely isolated (Figure 2E). 


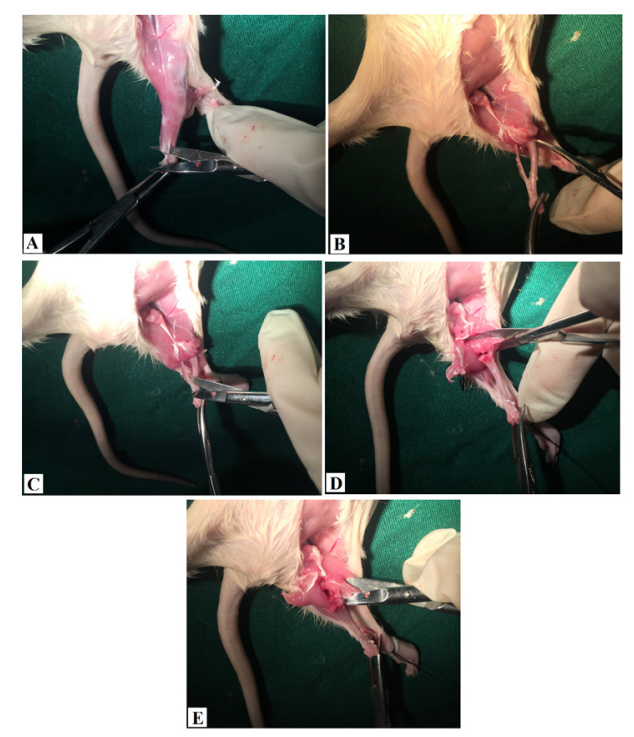

Figure 2. A. Dissection of ankle tendon. B. Isolation of tibia by sheer force of scissors. C. Dissection of fibula. D. and E. Twisting of the knee joint.

\section{Isolation of femurs}

Removal of the tibiae caused the patella and articular capsule of the knee joint (2-3 mm in length; Figure 3A) to be exposed. The articular capsule was tightly held by a pair of scissors and rotated toward the reversed genuflex of the knee joint, at which point the femoral capitulum was isolated (Figure 3B). The scissors were then slid further to the proximal end of the femur to reveal three quarters of the length of the femur (Figure $3 \mathrm{C}$ and D). The surrounding muscles attached to the head of the femur were then simply removed by pulling or cutting to obtain a clean femur (Figure 3E).

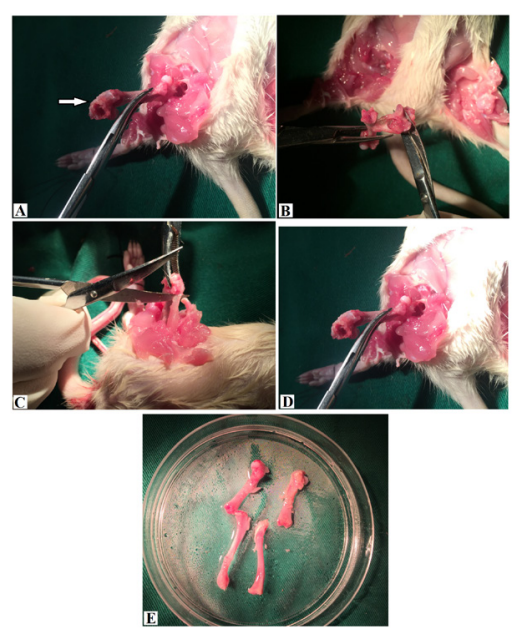

Figure 3. A. Patella (arrow). B. Isolation of femoral capitulum. C. Femur isolated by sheer force of the scissors. D. Cutting the remaining muscle tissue. E. Clean femurs and tibiae obtained. 


\section{Harvesting BMCs}

The isolated femurs and tibiae were immediately placed in a culture dish containing $10 \mathrm{~mL}$ cold $\left(4^{\circ}-10^{\circ} \mathrm{C}\right)$ PBS. The bones were washed twice with $5 \mathrm{~mL}$ cold PBS and 2-mm sections at both ends of the bones were cut to expose the bone marrow cavity. BMCs were flushed out using a syringe loaded with $8 \mathrm{~mL}$ cold RMPI 1640 and a $27 \mathrm{G}$ needle until the color of the bone marrow cavity turned pale (Figure $4 \mathrm{~A}$ and B). Occasionally, inadequate exposure of the bone marrow cavity caused substantial resistance against the insertion of the needle; this was remedied by further cutting the ends of the bone. The cell suspension was thoroughly blended by pipetting and filtering with a $38-\mu \mathrm{m}$ cell sieve. The cells were then inoculated into a $25-\mathrm{cm}^{2}$-flask and incubated at $37^{\circ} \mathrm{C}$, in a $5 \% \mathrm{CO}_{2}$ incubator at $90 \%$ humidity. Prior to the inoculation, a $100-\mu \mathrm{L}$ aliquot of the cell suspension was obtained using a burette, and the cells were counted in a blood counting chamber. Cells $\left(1 \times 10^{6}\right)$ were washed twice with ice-cold PBS and 5\% FBS and re-suspended in $500 \mu \mathrm{L}$ PBS and 5\% FBS. The cells were then analyzed using an Accuri C6 flow cytometer (BD Biosystems, Franklin Lakes, NJ, USA) and the CFlow Plus software. Five milliliter fresh culture medium was added after a 48 -h incubation; the cells were counted and analyzed using the flow cytometer again after $72 \mathrm{~h}$.

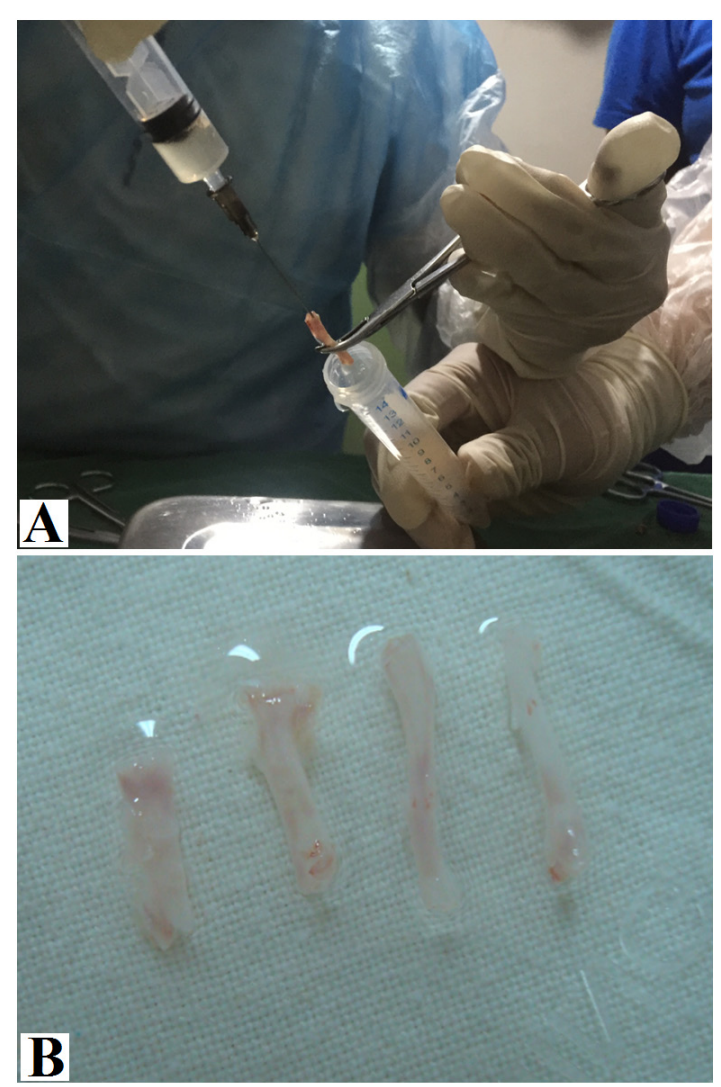

Figure 4. A. BMCs were flushed out of femurs and tibiae. B. Femurs and tibiae after flushing out the BMCs. 


\section{Statistical analyses}

All data are reported as means $\pm \mathrm{SD}$ (standard deviation). The differences among data were analyzed by analysis of variance (ANOVA) on SPSS v.16.0 (SPSS, Chicago, IL, USA). $P$ values $<0.05$ were considered to indicate statistically significant differences.

\section{RESULTS}

The efficiency and feasibility of the proposed method, carried out using a standard protocol with minimal changes, was tested on 21 rats. The bone dissection time was defined as the time from the dissection of the hind limbs to the acquisition of clean tibiae and femurs. The BMC suspension preparation time was defined as the time spent between euthanizing the rat and inoculation of the BMCs into the culture flask. Bone fractures or injuries to the bone marrow cavities were also recorded. The viable cell rates were determined before and after incubation for $72 \mathrm{~h}$ using the trypan blue staining assay. The cell suspensions were also microscopically examined to assess the contamination of BMCs by microbes, muscle cells, and fibroblasts.

The bone dissection and BMC suspension preparation times taken in this study were $1.8 \pm 0.6$ and $13.1 \pm 2.3$ min, respectively. We observed no unwarranted bone fractures or injuries to the bone marrow cavities during the procedure. Incubation for $72 \mathrm{~h}$ led to a significant increase in the cell number $\left(4.4 \pm 0.3 \times 10^{6}\right.$ to $\left.6.9 \pm 0.7 \times 10^{6} ; \mathrm{P}<0.01\right)$. However, the viable cell rates before and after incubation did not differ significantly $(98.1 \pm 0.6$ and $96.2 \pm 1.1 \%$, respectively; $\mathrm{P}>0.05$ ). The culture media incubated for 48 and $72 \mathrm{~h}$ assumed a lighter color; however, the culture media were not turbid, indicating the vigorous metabolism of the cultured cells. Microscopic examination revealed a $0 \%$ microbial contamination rate; moreover, no obvious muscle cell contamination was observed (Figure 5). Representative FCS-A plots of the BMCs cultured for $72 \mathrm{~h}$ exhibited favorable results compared to those of BMCs cultured for $0 \mathrm{~h}$ (Figure 6).

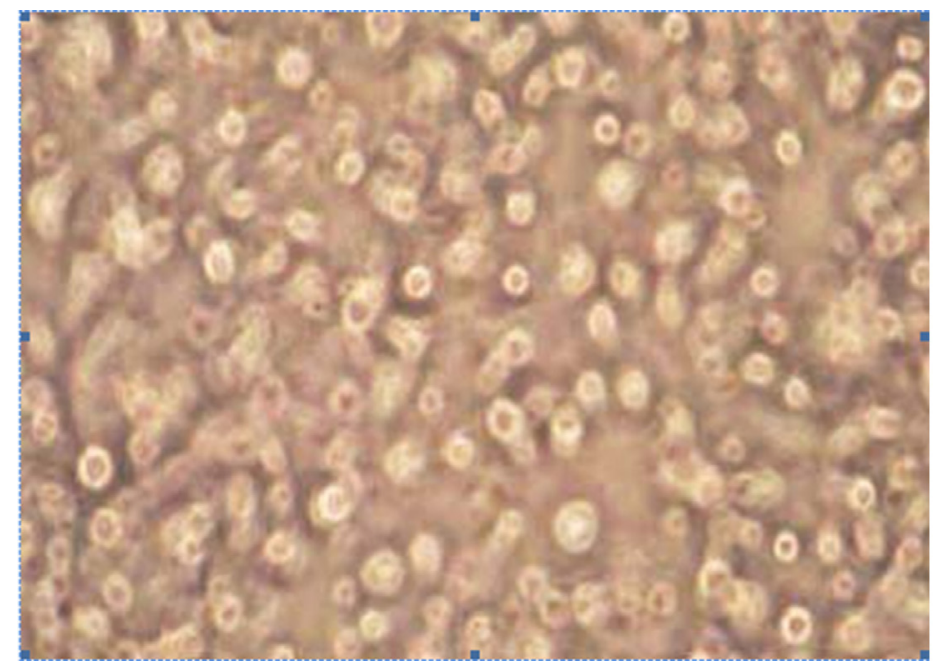

Figure 5. Observation of BMCs under a microscope after culturing for $72 \mathrm{~h}$ in RPMI 1640 medium (x100). 

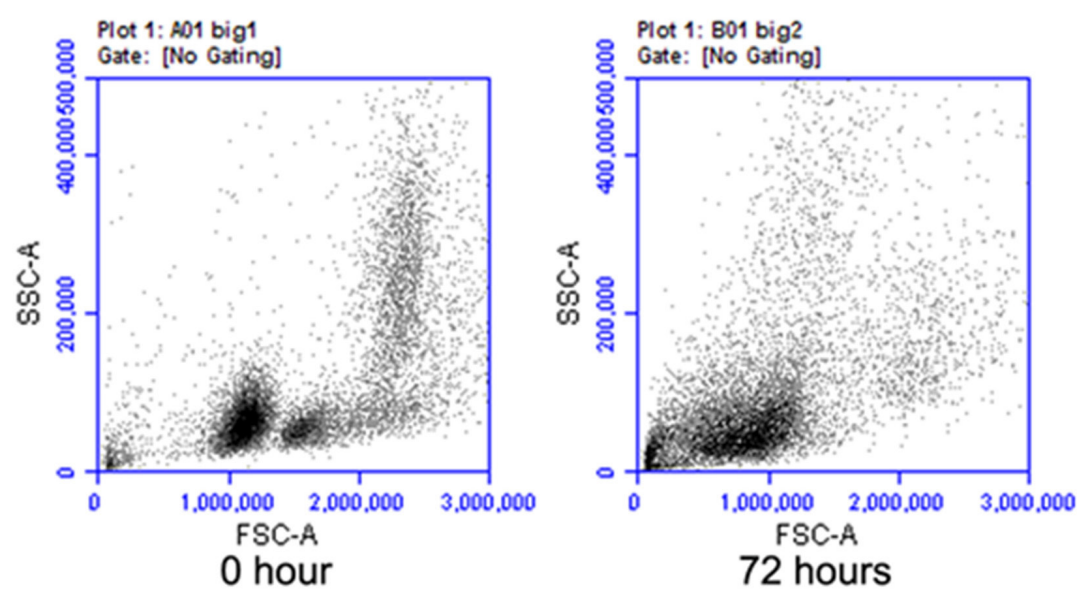

Figure 6. Flow cytometry analysis of the cultured BMCs.

\section{DISCUSSION}

A literature search revealed the lack of detailed descriptions of procedures used to extract clean, uncontaminated murine femurs and tibiae rapidly for BMC isolation. Moreover, the reported methods vary widely from investigator to investigator (Lutz et al., 1999; Ishii et al., 2005; Anjos-Afonso and Bonnet, 2008; Li et al., 2013). Some of these reported methods may appear to be simple; however, loose interpretations of these "simple" protocols, and wide variations in the practiced methods may have a significant impact on the ischemic time of BMCs. Li et al. (2013) detailed the extraction as follows: "the knee joint was first cut and then the muscle tissues surrounding femurs and tibias were removed by gauze, scalpel, or others". The use of these methods increases the susceptibility of the femur and tibia to mechanical damage; moreover, the time required to harvest BMCs from one rat is more than $30 \mathrm{~min}$. The anatomic structure of rat hind legs is such that the muscle tissues or tendons in the calves skip the ankle joint and are directly attached to the feet. Therefore, the skin of the calf is easily separated by making a 2-mm transection from the distal end of the ankle joint. Moreover, incomplete separation of the skin has been found to be favorable for the fixation of the hind limbs and subsequent operations. The muscle tissues surrounding the bones were removed by completely isolating the shafts of the tibia and fibula from the distal to the proximal ends of the bones using the shearing force of sliding scissors. There are numerous muscles attached to the knee joint, which increase the susceptibility of the tibia to fracture. In order to solve this problem when the fibula is revealed and cut, the scissors were slid further toward the proximal end above the knee joint, and the muscle was turned upward over the knee joint. The knee joint end of the tibia was then completely isolated and popped up by a pulley effect resulting from gentle twisting of the femur in the forward direction towards the anterior surface of the knee joint. The same procedure was used to isolate the femur rapidly, in order to preserve the integrity of the bone.

This method of holding the bone with forceps and using the three-step scissorbased "locate-slide-twist" procedure against the direction of muscle distribution allowed the complete removal of the muscles attached to the tibia and fibula. The use of this method has 
several advantages. Firstly, the method is quick and simple. Clean femurs and tibiae were obtained in only $1.9 \pm 0.4 \mathrm{~min}$, and in combination with direct washing of BMCs with RPMI 1640 medium without the centrifugation step, the entire preparation time was reduced to 14.7 \pm 1.8 min. Moreover, the influence of ischemic time on BMC viability could be minimized by typically performing this procedure on ice. However, the use of this method under normal conditions (without incubation on ice) also allowed for good BMC growth without a significant reduction in cell viability, even after a 72-h incubation period. This modified method also prevents contamination and injury to the bone marrow cavity. Finally, the tissues attached to the bone can be completely removed using this method. Complete removal of muscle and connective tissues is critical for the culture of bone marrow mesenchymal stem cells. Muscle cells can proliferate and contaminate the culture medium. Distinguishing muscle cells from mesenchymal stem cells based on the morphology is difficult; moreover, specific molecular markers for identification are currently unavailable (Ishii et al., 2005). As the muscles are isolated using the shearing force of scissors against the direction of muscle distribution, they can be completely removed by applying the requisite amount of pressure. In particular, twisting the knee joint end against the direction of the joint motion renders the metaphyses of the femurs and tibiae void of muscle tissue, obviating the need for the removal of muscles using blades, as described previously (Li et al., 2013).

In conclusion, we have developed a modified method for the isolation of BMCs from rat tibiae and femurs. This method can be used to isolate BMCs rapidly from rat tibiae and femurs without contamination from muscle cells or microbes, and with reduced incidence of bone marrow cavity injury. Furthermore, it may improve the influence of ischemic injury on BMCs by reducing the harvest time.

\section{Conflicts of interest}

The authors declare no conflict of interest.

\section{ACKNOWLEDGMENTS}

Research supported by the Health in Yunnan Province Research Fund Project (\#2012WS0078) and the Chinese Ministry of Education Doctoral Fund Project for New Teachers (\#4201002).

\section{REFERENCES}

Anjos-Afonso F and Bonnet D (2008). Isolation, culture, and differentiation potential of mouse marrow stromal cells. Curr. Protoc. Stem Cell Biol. Chapter 2: 3.

Davis BK (2013). Isolation, culture, and functional evaluation of bone marrow-derived macrophages. Methods Mol. Biol. 1031: 27-35. http://dx.doi.org/10.1007/978-1-62703-481-4_3

Ishii M, Koike C, Igarashi A, Yamanaka K, et al. (2005). Molecular markers distinguish bone marrow mesenchymal stem cells from fibroblasts. Biochem. Biophys. Res. Commun. 332: 297-303.

Li XY, Zhang Y and Qi GX (2013). Evaluation of isolation methods and culture conditions for rat bone marrow mesenchymal stem cells. Cytotechnology 65: 323-334.

Lutz MB, Kukutsch N, Ogilvie AL, Rössner S, et al. (1999). An advanced culture method for generating large quantities of highly pure dendritic cells from mouse bone marrow. J. Immunol. Methods 223: 77-92. http://dx.doi.org/10.1016/ $\underline{\text { S0022-1759(98)00204-X }}$

Smajilagić A, Aljičević M, Redžić A, Filipović S, et al. (2013). Rat bone marrow stem cells isolation and culture as a bone 
formative experimental system. Bosn. J. Basic Med. Sci. 13: 27-30.

Soleimani M and Nadri S (2009). A protocol for isolation and culture of mesenchymal stem cells from mouse bone marrow. Nat. Protoc. 4: 102-106. http://dx.doi.org/10.1038/nprot.2008.221

Talmor M, Mirza A, Turley S, Mellman I, et al. (1998). Generation or large numbers of immature and mature dendritic cells from rat bone marrow cultures. Eur. J. Immunol. 28: 811-817. http://dx.doi.org/10.1002/(SICI)15214141(199803)28:03<811::AID-IMMU811>3.0.CO;2-S 